\title{
ON THE CONSTRUCTION OF CODIMENSION-TWO MINIMAL IMMERSIONS OF EXOTIC SPHERES
}

\author{
BY WU-TEH HSIANG, WU-YI HSIANG AND IVAN STERLING
}

1. Introduction. It is, nowadays, a well-known fundamental fact in differential topology that there are many differential manifolds which are homeomorphic to but nondiffeomorphic to spheres. Such differentiable manifolds were christened exotic spheres by their founder, J. Milnor. The structures of those exotic spheres which can be realized as the boundaries of parallelizable manifolds were systematically analyzed by the technique of surgery in $[\mathbf{K M}]$. They can be imbedded as codimension-two submanifolds of the standard spheres.

In differential geometry and geometric measure theory, the study of closed minimal submanifolds of the euclidean $n$-sphere $S^{n}(1)$ is directly related to that of the local structure of singularities of minimal submanifolds in the general Riemannian setting. Therefore, closed minimal submanifolds of $S^{n}(1)$ are not only interesting, nice, geometric objects in themselves, but are also of basic, theoretical importance in the study of geometric variational theory. Among various problems on the existence, as well as on the uniqueness, of closed minimal submanifolds of euclidean spheres, the following problems naturally distinguish themselves as especially interesting.

Problem 1. The existence and uniqueness problem of minimal imbeddings (or immersions) of codimension-one spheres in $S^{n}(1)$ (the spherical Bernstein problem proposed by S. S. Chern [2]).

Problem 2. The existence and uniqueness problem of minimal imbeddings (or immersions) of codimension-two exotic or knotted spheres in $S^{n}(1)$.

We announce here the existence of codimension-two minimal immersions of exotic spheres into euclidean spheres. The following is a brief outline of a method of construction of such examples of minimal immersions of exotic spheres of Kervaire type, $\Sigma_{0}^{4 k+1}$, into $S^{4 k+3}(1)$.

2. The Basic ideas of construction. In the study of the degree of symmetry of exotic spheres [4], one finds that the Kervaire sphere $\Sigma_{0}^{4 k+1} \in$ $b P_{4 k+2}$ is the most symmetric exotic sphere, namely,

$$
\begin{aligned}
N\left(\Sigma^{m}\right) & =\max \left\{\operatorname{dim} G: G \text { compact subgroup of } \operatorname{Diff}\left(\Sigma^{m}\right)\right\} \\
& \leq \frac{1}{8}\left(m^{2}+7\right)
\end{aligned}
$$

for all exotic $m$-spheres $\Sigma^{m}$, and, in the above, equality holds when and only when $\Sigma^{m}=\Sigma_{0}^{4 k+1} \in b P_{4 k+2}$. Compact, differentiable transformation groups on $\Sigma_{0}^{4 k+1}$ with the above highest possible dimension, i.e., $\operatorname{dim} G=$ $\frac{1}{8}\left(m^{2}+7\right)=k(2 k+1)+1$, are classified in [3]. It is a rather pleasant surprise

Received by the editors July 26, 1984.

1980 Mathematics Subject Classification. Primary 53A10; Secondary 53C42. 
that $\operatorname{dim}\left(\Sigma_{0}^{4 k+1} / G\right)=1$, namely, the Kervaire sphere can be constructed as a generalized rotational manifold! Slightly later, Brieskorn [1] discovered that the Kervaire sphere $\Sigma_{0}^{4 k+1}$ can be imbedded into the unit sphere $S^{4 k+3}(1)$ as the solution of the following highly symmetric equation of complex variables:

$$
z_{0}^{l}+z_{1}^{2}+\cdots+z_{2 k+1}^{2}=0, \quad l \equiv \pm 3(\bmod 8) .
$$

Combining the above results of $[\mathbf{3}, \mathbf{4}]$, it is not difficult to see that those highest-dimensional compact transformation groups $\left(G, \Sigma_{0}^{4 k+1}\right)$ can, in fact, be equivariantly imbedded into the following orthogonal transformation groups on $S^{4 k+3}(1)$, respectively:

$$
G=S^{1} \times O(2 k+1) / \mathrm{Z}_{2}, \quad S^{4 k+3}(1) \subset \mathbb{C}^{1} \oplus \mathbb{C}^{2 k+1}=\mathbb{R}^{4 k+4},
$$

where the $O(2 k+1)$ part acts on $\mathbb{C}^{2 k+1}=\mathbb{R}^{2 k+1} \oplus \mathbb{R}^{2 k+1}$ diagonally via the standard $O(2 k+1)$-action on $\mathbb{R}^{2 k+1}$, and the $S^{1}$ part acts on $\mathbb{C}^{1} \oplus \mathbb{C}^{2 k+1}$ via componentwise complex multiplication of $\left(e^{2 i \theta}, e^{l i \theta}, \ldots, e^{l i \theta}\right)$.

Based upon the above background knowledge of the symmetries of Kervaire spheres, it is natural to try the following idea of constructing equivariant examples of minimal immersions of $\left(G, \Sigma_{0}^{4 k+1}\right)$ into $\left(G, S^{4 k+3}(1)\right)$ with respect to the above specific transformation groups. The actual construction of such equivariant examples consists of the following steps:

Step 1. One begins with the determination of the orbital geometry of the above specific equivariant geometric system $\left(G, S^{4 k+1}(1)\right)$. For our purpose one needs the determination of the orbital distance metric on the orbit space $S^{4 k+3}(1) / G$, which measures the distance between orbits, and the volume function $v(\xi)$, which records the (4k-dimensional) volume or the orbits $\xi \in$ $S^{4 k+3}(1) / G$. This groundwork has already been done in a previous paper [5] by the first two authors. The results can be summarized as follows:

(i) Topologically, the orbit space is a 3-dimensional disc $D^{3}$ with a natural induced action of $O(2)=N(G, O(4 k+2)) / G$. The orbital geometric structure on $D^{3}$ is clearly $O(2)$-invariant.

(ii) Choose a fixed subgroup $O(1) \subset O(2)$. Let $D^{2}=F\left(O(1), D^{3}\right)=$ $D_{+}^{2} \cup D_{-}^{2}$. Then $D_{+}^{2}$ (or $\left.D_{-}^{2}\right)$ forms a fundamental domain of $\left(O(2), D^{3}\right)$, and the restricted orbital geometry on $D_{+}^{2}$ can be described as follows.

It is convenient to parametrize $D_{+}^{2}$ by $\{(x, y) ; 0 \leq x \leq \pi / 2,0 \leq y \leq \pi / 4\}$. Then the orbital distance metric and the volume function, restricted to $D_{+}^{2}$, can simply be given by the following formula:

$$
\begin{gathered}
d s^{2}=d x^{2}+\cos ^{2} x d y^{2}, \\
v^{2}(x, y)=c \cdot\left\{\sin ^{2} 2 x+l^{2} \cos ^{4} x \sin ^{2} 2 y\right\} \cdot\left\{\cos ^{4} x \cos ^{2} 2 y\right\}^{n-2} .
\end{gathered}
$$

Step 2. Following the setting of $[\mathbf{H L}]$, it is convenient to modify the orbital distance metric $d s^{2}$ on the orbit space $D^{3}=S^{4 k+3}(1) / G$ by setting $d \bar{s}^{2}=$ $v^{2} \cdot d s^{2}$. Then an invariant submanifold $\left(G, M^{4 k+1}\right)$ of cohomogeneity one in $\left.\left(G, S^{4 k+3}\right)(1)\right)$ is minimal if and only if its generating curve $M^{4 k+1} / G$ is a geodesic curve with respect to the above modified metric $d \bar{s}^{2}$. Since $D^{2}=F\left(O(1), D^{3}\right)$ is the fixed point set of an isometric transformation group, it is clearly a totally geodesic submanifold of $\left(D^{3}, d \bar{s}^{2}\right)$. This remarkable, 


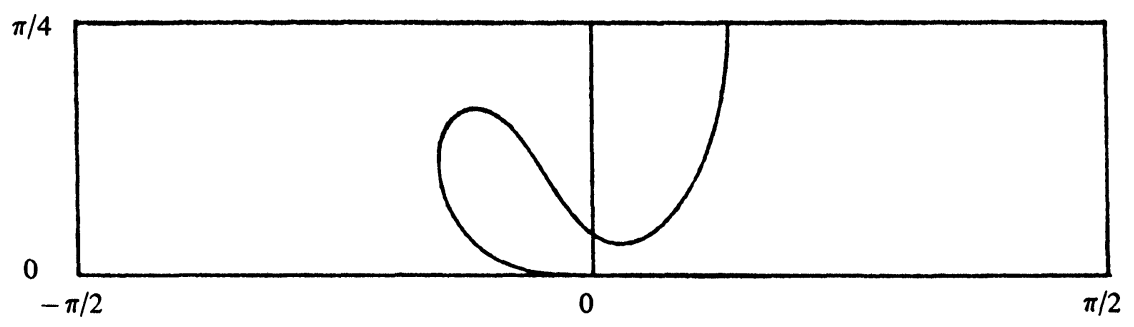

FIGURE 1

simple fact enables us to reduce the original codimension-two problem to a much simpler codimension-one problem. Analytically, this amounts to a reduction from a system of two nonlinear second order ODEs to that of a single ODE, namely,

$$
\begin{aligned}
\cos x \cdot\left(x^{\prime} y^{\prime \prime}-y^{\prime} x^{\prime \prime}\right)-\sin x \cdot\left(2 x^{\prime 2}\right. & \left.+y^{\prime 2} \cdot \cos x\right) \cdot y^{\prime} \\
+\cos x\left(x^{\prime 2}+y^{\prime 2} \cos ^{2} x\right) y^{\prime}\{ & -2(n-2) \tan x \\
& \left.+\frac{\sin 4 x-2 l^{2} \cos ^{3} x \sin x \sin ^{2} 2 y}{\sin ^{2} 2 x+l^{2} \cos ^{4} x \sin ^{2} 2 y}\right\} \\
-\frac{x^{\prime}}{\cos x}\left(x^{\prime 2}+y^{\prime 2} \cos ^{2} x\right)\{-2(n-2) \tan 2 y & \left.-\frac{l^{2} \cos ^{4} x \sin 4 y}{\sin ^{2} 2 x+l^{2} \cos ^{4} x \sin ^{2} 2 y}\right\} \\
=0, &
\end{aligned}
$$

where the "geodesic" curve $\gamma(t)=(x(t), y(t)),|x| \leq \pi / 4,|y| \leq \pi / 4$, is given in terms of an arbitrary parameter $t$ and $x^{\prime}, y^{\prime}, x^{\prime \prime}, y^{\prime \prime}$ are derivatives with respect to $t$.

Step 3. Of course, the most crucial step of the construction is to establish the existence of a global solution curve of the above ODE (2) with correct geometric features which will generate an immersed Kervaire sphere under the action of $G$. In our case it is a solution curve which starts at a suitable boundary point $(a, \pi / 4), 0<a<\pi / 2$, and ends up at the origin. Figure 1 is the computer-generated graph of such a curve for the case $l=3, n=2 k+1=$ 5 .

\section{Statement of the result and an indication of its proof.}

THEOREM. For $k \leq 100$ there exists a minimal immersion of the Kervaire sphere $\Sigma_{0}^{4 k+1}$ into $S^{4 k+3}(1)$ which is of cohomogeneity one.

Sketch of Proof. As described in $\S 2$, within the framework of equivariant Riemannian geometry, the proof may be reduced to the construction of a global solution curve of the ODE (2) with the correct geometric features. Let $\gamma(x, y, \theta)$ be the solution curve starting at $(x, y)$ with initial angle $\theta$ with 


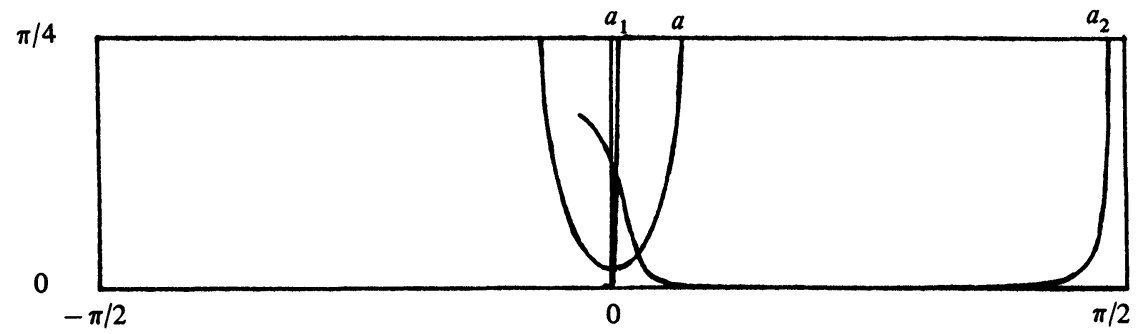

FIGURE 2

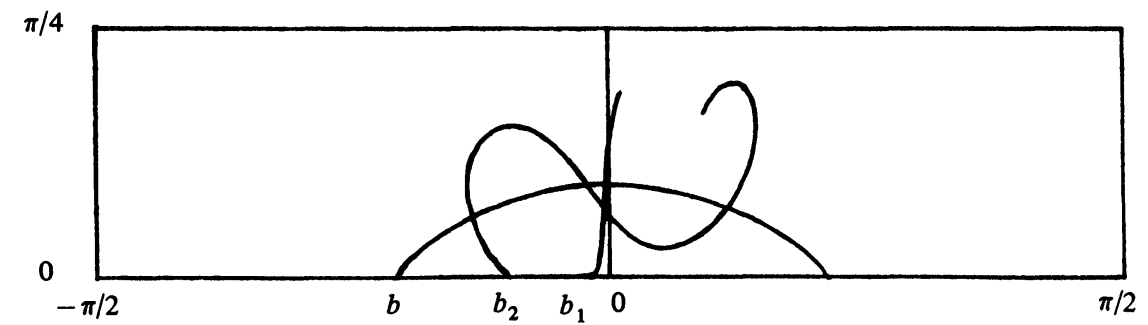

FIGURE 3

$\partial / \partial x$. The following facts highlight a stagewise evolution through solution curves leading to the desired one.

FACT 1. There exist $a_{1}, a_{2}, 0<a_{1}, a_{2}<\pi / 2$, such that $\gamma\left(a_{1}, \pi / 4,-\pi / 2\right)$ (resp., $\gamma\left(a_{2}, \pi / 4,-\pi / 2\right)$ ) "intersects" $x \equiv 0$ with an angle $\alpha, 0<\alpha<\pi / 2$ (resp., $\pi / 2<\alpha<\pi$ ). Such curves are shown to exist by studying the Jacobi equation along the known geodesics $x \equiv 0$ and $y \equiv 0$.

FACT 2. There exists $a, 0<a<\pi / 2$, such that $\gamma(a, \pi / 4,-\pi / 2)$ "intersects" $x \equiv 0$ orthogonally. See Figure 2 .

FACT 3. There exist $b_{1}, b_{2}, B_{1}, B_{2}, \pi / 2<b_{1}, b_{2}<0,0<B_{1}, B_{2}<\pi$, such that $\gamma\left(b_{1}, 0, B_{1}\right)$ ) "intersects" $x=0$ with an angle $\alpha, 0<\alpha<\pi / 2$ (resp., $\pi / 2<\alpha<\pi$ ). Furthermore, $\gamma\left(b_{2}, 0, B_{2}\right)$ has $d y / d s>0$ at its "first turning back point" The curve $\gamma\left(b_{2}, 0, B_{2}\right)$ is shown to exist, with the aid of a computer, by using numerical approximations.

FACT 4. There exist $b, B,-\pi / 2<b<0,0<B<\pi$, such that $\gamma(b, 0, B)$ "intersects" $x \equiv 0$ orthogonally. See Figure 3 .

FACT 5. There exist $d_{1}, \delta_{1},-\pi / 2<d_{1}<0,0<\delta_{1}<\pi$, such that $\left(d_{1}, 0, \delta_{1}\right)$ goes through the origin. See Figure 4.

FACT 6. There exist $d_{2}, \delta_{2},-\pi / 2<d_{2}<0,0<\delta_{2}<\pi$, such that $\gamma\left(d_{2}, 0, \delta_{2}\right)$ "intersects" $y \equiv \pi / 4$. 


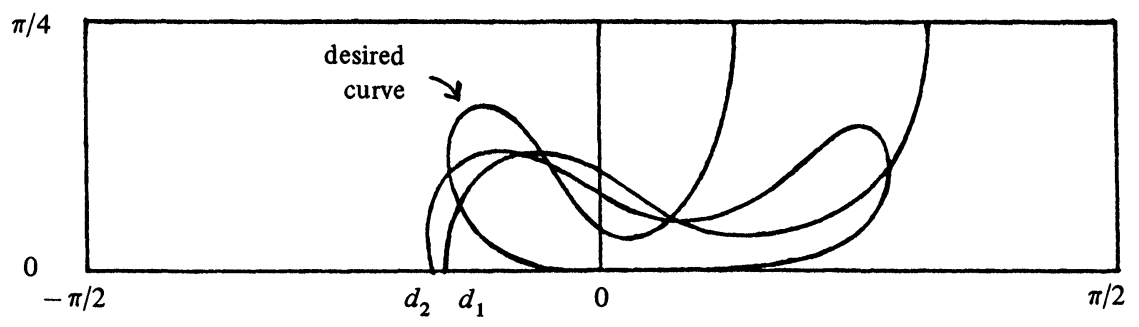

FIGURE 4

The Theorem follows from Facts 2 and 6 and continuous dependence of solutions on initial conditions.

\section{REFERENCES}

[KM] M. Kervaire and J. Milnor, Groups of homotopy spheres. I, Ann. of Math. (2) 77 (1963), 504-537.

[HL] W. Y. Hsiang and B. H. Lawson, Jr., Minimal submanifolds of low cohomogeneity, J. Differential Geom. 5 (1971), 1-38.

[1] E. Brieskorn, Examples of singular normal complex space which are topological manifolds, Proc. Nat. Acad. Sci. U.S.A. 55 (1966), 1395-1397.

[2] S. S. Chern, Differential geometry, its past and its future, Actes Congres Intern. Math., 1970, Tome 1, pp. 41-53.

[3] W. C. Hsiang and W. Y. Hsiang, On compact subgroups of the diffeomorphism groups of Kervaire spheres, Ann. of Math. (2) 85 (1967), 359-369.

[4] W. Y. Hsiang, On the bound of the dimensions of the isometry groups of all possible Riemannian metrics on an exotic sphere, Ann. of Math. (2) 85 (1967), 351-358.

[5] W. T. Hsiang and W. Y. Hsiang, On the construction of constant mean curvature imbeddings of exotic and/or knotted spheres into the unit sphere. III (to appear).

[6] J. Milnor, On manifolds homeomorphic to the 7-sphere, Ann. of Math. (2) 64 (1956), $399-405$.

Department of Mathematics, Syracuse University, Syracuse, NeW YORK 13210 (Current address of Wu-teh Hsiang)

Department OF MATHEMATICS, UNIVERSity OF CALIFORNiA, BERKELEy, CALIFORNIA 94720 (Current address of Wu-yi Hsiang and I. Sterling) 